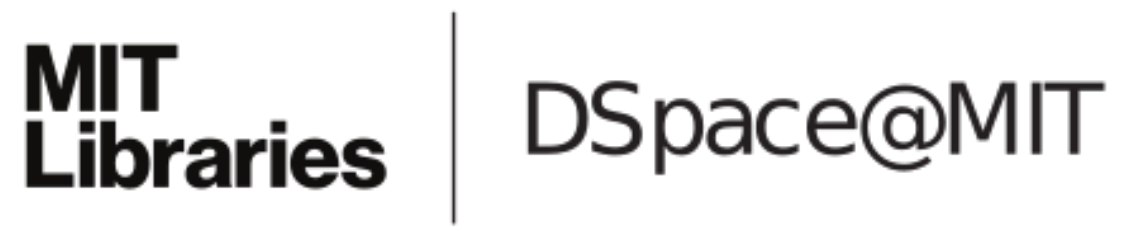

\author{
MIT Open Access Articles
}

Theory of thermal emission from periodic structures

The MIT Faculty has made this article openly available. Please share how this access benefits you. Your story matters.

Citation: Han, S. E. "Theory of thermal emission from periodic structures." Physical Review B 80.15 (2009): 155108. (c) 2009 The American Physical Society

As Published: http://dx.doi.org/10.1103/PhysRevB.80.155108

Publisher: American Physical Society

Persistent URL: http://hdl.handle.net/1721.1/52485

Version: Final published version: final published article, as it appeared in a journal, conference proceedings, or other formally published context

Terms of Use: Article is made available in accordance with the publisher's policy and may be subject to US copyright law. Please refer to the publisher's site for terms of use. 


\title{
Theory of thermal emission from periodic structures
}

\author{
S. E. Han* \\ Department of Chemical Engineering and Materials Science, University of Minnesota, Minneapolis, Minnesota 55455, USA
}

(Received 22 May 2009; revised manuscript received 3 August 2009; published 5 October 2009)

\begin{abstract}
We provide a momentum space formulation for thermal emission from photonic crystals and corroborate Kirchhoff's law for periodic structures. This formalism allows us to calculate the optical coherence in the far and near fields of photonic crystals. Calculations in the far field show that the exchange of momentum between a linear grating and the surface polaritons can significantly decrease the coherence length for the grating compared to that of a flat surface. Considerations in the near field of photonic crystals show that, unlike observations of flat surfaces, the electric energy density does not necessarily decrease as $1 / z^{3}$ for a distance $z$ from the surface.
\end{abstract}

DOI: $10.1103 /$ PhysRevB.80.155108

PACS number(s): 42.70.Qs, 44.40.+a, 73.20.Mf

\section{INTRODUCTION}

Control of thermal emission is essential for various applications including lighting, ${ }^{1}$ imaging, ${ }^{2}$ and heat-to-electrical conversion systems such as in thermophotovoltaics. ${ }^{3,4}$ Thermal emission can be dramatically modified when the heated objects are periodically structured on an optical length scale. ${ }^{5-7}$ In these structures, known as photonic crystals, ${ }^{8}$ optical diffraction leads to a modification of the photonic density of states. This affects the thermal emission.

A recent experimental study reported that thermal emission from a photonic crystal could exceed Planck's blackbody limit. ${ }^{3}$ However, it was subsequently argued that this would violate the second law of thermodynamics. ${ }^{9}$ This argument assumed thermal equilibrium between the photonic crystal and the radiation field. Consequently, a potential explanation for the experiments was that they represented the effect of nonequilibrium processes. Out of equilibrium, the emission could potentially be higher than the black body. ${ }^{10-12}$ A theoretical study ${ }^{13}$ dealt with a nonequilibrium thermal emission where a photonic crystal is emitting into vacuum. Specifically, the photonic crystal is not in equilibrium with the electromagnetic field but equilibrium is assumed to be achieved inside the material. In this case, calculations predicted not only that the black-body limit should be maintained but also that Kirchhoff's law should be obeyed. Subsequent experiments supported this conclusion. ${ }^{14,15}$ In fact, before photonic crystals were introduced, Kirchhoff's law had already been proved for the more general case of any arbitrary scattering object. ${ }^{16,17}$ This suggests that any periodic structures should obey Kirchhoff's law.

In this study, we provide a momentum space formulation within the framework of fluctuational electrodynamics for the proof of Kirchhoff's law for periodic structures. The formalism used can also be extended to optical coherence. Using the formalism, we investigate the optical coherence in the far and near fields of a periodic structure. We also show that the behavior of the electric energy density in the near field of a photonic crystal is different from a flat surface. For example, at a distance $z$ from the surface, the electric energy density does not necessarily scale as $1 / z^{3}$ in the near field, as is the case for a flat surface. ${ }^{17,18}$

\section{CALCULATING GREEN'S DYADIC FROM THE TRANSFER MATRIX}

In the formulation of fluctuational electrodynamics, we will use the Green's dyadic which relates a point source to the resulting fields. Because many methods for calculating electromagnetic fields for periodic structures generate transmission and reflection coefficients, it is useful to obtain the Green's dyadic in terms of these optical coefficients. A matrix constructed from the optical coefficients for the plane waves of different wave vectors and polarizations is called the transfer matrix. In this section, we will find a relation between the Green's dyadic and the transfer matrix.

Consider light thermally emitted into vacuum from a periodically structured film. For simplicity, we assume that the film is composed of nonmagnetic materials, lies in the $x y$ plane, and extends in $z$ from $-L$ to 0 . Due to the periodicity in the $x y$ plane, Bloch waves will be developed. Thus, the fields can be represented by the sum of the Bloch waves as

$$
\boldsymbol{V}\left(\boldsymbol{r}_{\|}, z, \omega\right)=\sum_{\boldsymbol{k}_{\|}} \boldsymbol{V}_{\boldsymbol{k}_{\|}}\left(\boldsymbol{r}_{\|}, z, \omega\right),
$$

where $\boldsymbol{V}$ is a field, $\boldsymbol{r}_{\|}$and $\boldsymbol{k}_{\|}$are the position and the wave vectors parallel to the surface ( $x y$ plane), $\boldsymbol{V}_{\boldsymbol{k}_{\|}}$is the Bloch wave with the wave vector component $\boldsymbol{k}_{\|}$, and $\omega$ is the angular frequency. Note that $\boldsymbol{k}_{\|}$lies in the first Brillouin zone and takes discrete values because the size of the structured film is finite in the $x y$ plane. The Bloch wave $\boldsymbol{V}_{\boldsymbol{k}_{\|}}$should satisfy the Bloch theorem

$$
\boldsymbol{V}_{\boldsymbol{k}_{\|}}\left(\boldsymbol{r}_{\|}+\boldsymbol{a}_{\|}, z, \omega\right)=\boldsymbol{V}_{\boldsymbol{k}_{\|}}\left(\boldsymbol{r}_{\|}, z, \omega\right) e^{i \boldsymbol{k}_{\|} \cdot \boldsymbol{a}_{\|}},
$$

where $\boldsymbol{a}_{\|}$is the lattice vector in the $x y$ plane. If we expand the Bloch wave using the reciprocal-lattice vector $\boldsymbol{g}_{\|}$as

$$
\boldsymbol{V}_{\boldsymbol{k}_{\|}}\left(\boldsymbol{r}_{\|}, z, \omega\right)=\sum_{\boldsymbol{g}_{\|}} \tilde{\boldsymbol{V}}\left(\boldsymbol{k}_{\|}+\boldsymbol{g}_{\|}, z, \omega\right) e^{i\left(\boldsymbol{k}_{\|}+\boldsymbol{g}_{\|}\right) \cdot \boldsymbol{r}_{\|}},
$$

the Bloch theorem [Eq. (2)] is satisfied because

$$
e^{i g_{\|} \cdot a_{\|}}=1 \text {. }
$$

$\widetilde{\boldsymbol{V}}$ can be obtained by the two-dimensional inverse Fourier transform of Eq. (3) as 


$$
\tilde{\boldsymbol{V}}\left(\boldsymbol{k}_{\|}+\boldsymbol{g}_{\|}, z, \omega\right)=\frac{1}{S_{u c}} \int_{u c} d \boldsymbol{r}_{\|} \boldsymbol{V}_{\boldsymbol{k}_{\|}}\left(\boldsymbol{r}_{\|}, z, \omega\right) e^{-i\left(\boldsymbol{k}_{\|}+\boldsymbol{g}_{\|}\right) \cdot \boldsymbol{r}_{\|},}
$$

where $u c$ stands for the two-dimensional unit cell in the $x y$ plane and $S_{u c}$ is the surface area of the unit cell. Substituting Eq. (3) into Eq. (1), we have

$$
\boldsymbol{V}\left(\boldsymbol{r}_{\|}, z, \omega\right)=\sum_{\boldsymbol{k}_{\|}} \sum_{\boldsymbol{g}_{\|}} \tilde{\boldsymbol{V}}\left(\boldsymbol{k}_{\|}+\boldsymbol{g}_{\|}, z, \omega\right) e^{i\left(\boldsymbol{k}_{\|}+\boldsymbol{g}_{\|}\right) \cdot \boldsymbol{r}_{\|}} .
$$

Therefore $\tilde{\boldsymbol{V}}$ can also be obtained by the inverse Fourier transform of Eq. (6) as

$$
\tilde{\boldsymbol{V}}\left(\boldsymbol{k}_{\|}+\boldsymbol{g}_{\|}, z, \omega\right)=\frac{1}{S} \int d \boldsymbol{r}_{\|} \boldsymbol{V}\left(\boldsymbol{r}_{\|}, z, \omega\right) e^{-i\left(\boldsymbol{k}_{\|}+\boldsymbol{g}_{\|}\right) \cdot \boldsymbol{r}_{\|},}
$$

where $S$ is the surface area of the film.

Consider the electric field in vacuum that results from the thermal fluctuations in the electric current $j$ inside the structure. They are related to each other through the Green's dyadic $G$ as

$$
\begin{aligned}
E_{l}\left(\boldsymbol{r}_{\|}, z, \omega\right)= & i \omega \mu_{0} \int d \boldsymbol{r}_{\|}^{\prime} \int d z^{\prime} \\
& \times G_{l m}\left(\boldsymbol{r}_{\|}, \boldsymbol{r}_{\|}^{\prime} ; z, z^{\prime} ; \omega\right) j_{m}\left(\boldsymbol{r}_{\|}^{\prime}, z^{\prime}, \omega\right),
\end{aligned}
$$

where the subscripts $l, m=x, y, z$ and $\mu_{0}$ is the permeability of free space. The Green's dyadic $\stackrel{\leftrightarrow}{G}\left(\boldsymbol{r}_{\|}, \boldsymbol{r}_{\|}^{\prime} ; z, z^{\prime} ; \omega\right)$ relates the electric current at position $\left(\boldsymbol{r}_{\|}^{\prime}, z^{\prime}\right)$ to the electric field at position $\left(\boldsymbol{r}_{\|}, z\right)$ at frequency $\omega$. The Green's dyadic is known to satisfy 19

$$
\overleftrightarrow{G}^{T}\left(\boldsymbol{r}_{\|}, \boldsymbol{r}_{\|}^{\prime} ; z, z^{\prime} ; \omega\right)=\overleftrightarrow{G}\left(\boldsymbol{r}_{\|}^{\prime}, \boldsymbol{r}_{\|} ; z^{\prime}, z ; \omega\right) .
$$

Equation (9) is the consequence of the electrodynamic reciprocity theorem. ${ }^{17,20}$ This theorem roughly states that a fluctuating current and the electric field that it creates at a distant location can be interchanged without affecting their relationship. If we consider a point current in Eq. (8) and compare this to the relationship when the current and the field are interchanged, we can obtain Eq. (9) from the reciprocity theorem. Substituting Eq. (6) for $\boldsymbol{V}=\boldsymbol{j}$ into Eq. (8) and the resulting equation into Eq. (7) for $\boldsymbol{V}=\boldsymbol{E}$, we have

$$
\begin{aligned}
\widetilde{E}_{l}\left(\boldsymbol{k}_{\|}+\boldsymbol{g}_{\|}, z, \omega\right)= & \sum_{\boldsymbol{k}_{\|}^{\prime}, \boldsymbol{g}_{\|}^{\prime}} \int d z^{\prime} \times G_{l m}\left(\boldsymbol{k}_{\|}+\boldsymbol{g}_{\|}, \boldsymbol{k}_{\|}^{\prime}\right. \\
& \left.+\boldsymbol{g}_{\|}^{\prime} ; z, z^{\prime} ; \omega\right) \widetilde{j}_{m}\left(\boldsymbol{k}_{\|}^{\prime}+\boldsymbol{g}_{\|}^{\prime}, z^{\prime}, \omega\right),
\end{aligned}
$$

where

$$
\begin{aligned}
& G_{l m}\left(\boldsymbol{k}_{\|}+\boldsymbol{g}_{\|}, \boldsymbol{k}_{\|}^{\prime}+\boldsymbol{g}_{\|}^{\prime} ; z, z^{\prime} ; \omega\right) \\
& \quad=\frac{i \omega \mu_{0}}{S} \int d \boldsymbol{r}_{\|} \int d \boldsymbol{r}_{\|}^{\prime} \\
& \quad \times G_{l m}\left(\boldsymbol{r}_{\|}, \boldsymbol{r}_{\|}^{\prime} ; z, z^{\prime} ; \omega\right) e^{i\left[\left(\boldsymbol{k}_{\|}^{\prime}+\boldsymbol{g}_{\|}^{\prime}\right) \cdot \boldsymbol{r}_{\|}^{\prime}\left(\boldsymbol{k}_{\|}+\boldsymbol{g}_{\|}\right) \cdot \boldsymbol{r}_{\|}\right]} .
\end{aligned}
$$

In Eq. (10), $\tilde{\boldsymbol{E}}$ and $\tilde{\boldsymbol{j}}$ should have the same $\boldsymbol{k}_{\|}$because of the conservation of parallel momentum. Thus the Green's dyadic satisfies
$G_{l m}\left(\boldsymbol{k}_{\|}+\boldsymbol{g}_{\|}, \boldsymbol{k}_{\|}^{\prime}+\boldsymbol{g}_{\|}^{\prime} ; z, z^{\prime} ; \omega\right)=G_{l m}\left(\boldsymbol{k}_{\|}+\boldsymbol{g}_{\|}, \boldsymbol{k}_{\|}+\boldsymbol{g}_{\|}^{\prime} ; z, z^{\prime} ; \omega\right) \delta_{\boldsymbol{k}_{\|}, \boldsymbol{k}_{\|}^{\prime}}$

and Eq. (10) becomes

$$
\begin{aligned}
\widetilde{E}_{l}\left(\boldsymbol{k}_{\|}+\boldsymbol{g}_{\|}, z, \omega\right)= & \sum_{\boldsymbol{g}_{\|}^{\prime}} \int d z^{\prime} \times G_{l m}\left(\boldsymbol{k}_{\|}+\boldsymbol{g}_{\|}, \boldsymbol{k}_{\|}\right. \\
& \left.+\boldsymbol{g}_{\|}^{\prime} ; z, z^{\prime} ; \omega\right) \tilde{j}_{m}\left(\boldsymbol{k}_{\|}+\boldsymbol{g}_{\|}^{\prime}, z^{\prime}, \omega\right) .
\end{aligned}
$$

The Green's dyadic $\stackrel{\leftrightarrow}{G}\left(\boldsymbol{k}_{\|}+\boldsymbol{g}_{\|}, \boldsymbol{k}_{\|}+\boldsymbol{g}_{\|}^{\prime} ; z, z^{\prime} ; \omega\right)$ relates the electric current component of parallel wave vector $\boldsymbol{k}_{\|}+\boldsymbol{g}_{\|}^{\prime}$ at the $z=z^{\prime}$ plane to the electric field component of parallel wave vector $\boldsymbol{k}_{\|}+\boldsymbol{g}_{\|}$at the $z=z$ plane at frequency $\omega$. Taking the transpose of Eq. (11) and using Eqs. (9) and (12), we have

$$
\overleftrightarrow{G}^{T}\left(\boldsymbol{k}_{\|}+\boldsymbol{g}_{\|}, \boldsymbol{k}_{\|}+\boldsymbol{g}_{\|}^{\prime} ; z, z^{\prime} ; \omega\right)=\overleftrightarrow{G}\left(-\boldsymbol{k}_{\|}-\boldsymbol{g}_{\|}^{\prime},-\boldsymbol{k}_{\|}-\boldsymbol{g}_{\|} ; z^{\prime}, z ; \omega\right) \text {. }
$$

$\overleftrightarrow{G}\left(\boldsymbol{r}_{\|}, \boldsymbol{r}_{\|}^{\prime} ; z, z^{\prime} ; \omega\right)$ can be expressed by taking the Fourier transform of Eq. (11) and using Eq. (14) as

$$
\begin{aligned}
G_{l m}\left(\boldsymbol{r}_{\|}, \boldsymbol{r}_{\|}^{\prime} ; z, z^{\prime} ; \omega\right) & \\
= & \frac{1}{i \omega \mu_{0} S} \sum_{\boldsymbol{k}_{\|}} \sum_{\boldsymbol{g}_{\|, \boldsymbol{g}_{\|}^{\prime}}^{\prime}} G_{m l}\left(-\boldsymbol{k}_{\|}-\boldsymbol{g}_{\|}^{\prime},-\boldsymbol{k}_{\|}-\boldsymbol{g}_{\|} ; z^{\prime}, z ; \omega\right) \\
& \times e^{i\left(\boldsymbol{k}_{\|}+\boldsymbol{g}_{\|}\right) \cdot \boldsymbol{r}_{\|}-i\left(\boldsymbol{k}_{\|}+\boldsymbol{g}_{\|}^{\prime}\right) \cdot \boldsymbol{r}_{\|}^{\prime}} .
\end{aligned}
$$

To consider the polarization property of thermal emission, we define $s$ - and $p$-polarization unit vectors as ${ }^{21}$

$$
\begin{gathered}
\hat{\boldsymbol{s}}_{\boldsymbol{q}_{\|}, \pm} \equiv \hat{\boldsymbol{q}}_{\|} \times \hat{z}, \\
\hat{\boldsymbol{p}}_{\boldsymbol{q}_{\|, \pm}} \equiv \frac{c}{\omega}\left[\mp k_{z, v}\left(\boldsymbol{q}_{\|}, \omega\right) \hat{\boldsymbol{q}}_{\|}+\left|\boldsymbol{q}_{\|}\right| \hat{z}\right],
\end{gathered}
$$

where $\boldsymbol{q}_{\|}=\boldsymbol{k}_{\|}+\boldsymbol{g}_{\|}, \hat{\boldsymbol{q}}_{\|}=\boldsymbol{q}_{\|} / \boldsymbol{q}_{\|} \mid$, and + and - indicate whether the light is propagating or evanescent in the $+z$ and $-z$ directions, respectively. In Eq. (16), $k_{z, v}$ is the $z$ component of the wave vector in vacuum and is obtained by

$$
k_{z, v}\left(\boldsymbol{k}_{\|}+\boldsymbol{g}_{\|,}, \omega\right)=\sqrt{\frac{\omega^{2}}{c^{2}}-\left|\boldsymbol{k}_{\|}+\boldsymbol{g}_{\|}\right|^{2}},
$$

and $k_{z, v}$ is taken such that its imaginary part is positive but, if the imaginary part vanishes, the real part is non-negative. This sign convention is chosen to ensure that the wave is propagating or evanescent in the $\pm z$ direction for the \pm sign in Eq. (16).

To relate the Green's dyadic to the transfer matrix, consider an electric field in vacuum at the $z>0$ plane generated by a current at the same plane. This electric field incident on the structure is ${ }^{21}$

$$
\begin{aligned}
d \widetilde{E}_{i n c, l}( & \left.-\boldsymbol{k}_{\|}-\boldsymbol{g}_{\|,},, \omega\right) \\
= & -\frac{\omega \mu_{0}}{2 k_{z, v}\left(\boldsymbol{k}_{\|}+\boldsymbol{g}_{\|,}, \omega\right)} \\
& \quad \times \sum_{\alpha} \hat{\alpha}_{-\boldsymbol{k}_{\|} \boldsymbol{g}_{\|,}} \hat{\alpha}_{-\boldsymbol{k}_{\|}-\boldsymbol{g}_{\|}, m} \tilde{j}_{m}\left(-\boldsymbol{k}_{\|}-\boldsymbol{g}_{\|,}, z, \omega\right) d z,
\end{aligned}
$$


where the subscript inc denotes incident light and $\hat{\boldsymbol{\alpha}}=\hat{\boldsymbol{s}}_{-}$or $\hat{\boldsymbol{p}}_{-}$. The electric field at a different plane $z^{\prime}<0$ with parallel wave vector $-\boldsymbol{k}_{\|}-\boldsymbol{g}_{\|}^{\prime}$ resulting from the incident wave is expressed in terms of the transfer matrix $\stackrel{M}{\text { by }}$

$$
\begin{aligned}
d \widetilde{E}_{l}\left(-\boldsymbol{k}_{\|}-\boldsymbol{g}_{\|}^{\prime}, z^{\prime}, \omega\right)= & \sum_{\boldsymbol{g}_{\|}} M_{l m}\left(-\boldsymbol{k}_{\|}-\boldsymbol{g}_{\|}^{\prime},-\boldsymbol{k}_{\|}-\boldsymbol{g}_{\|} ; z^{\prime}, z ; \omega\right) \\
& \times d \tilde{E}_{i n c, m}\left(-\boldsymbol{k}_{\|}-\boldsymbol{g}_{\|}, z, \omega\right)
\end{aligned}
$$

Plugging Eq. (18) into Eq. (19) and comparing the resulting equation with

$$
\begin{aligned}
d \widetilde{E}_{l}\left(-\boldsymbol{k}_{\|}-\boldsymbol{g}_{\|}^{\prime}, z^{\prime}, \omega\right)= & \sum_{\boldsymbol{g}_{\|}} G_{l m}\left(-\boldsymbol{k}_{\|}-\boldsymbol{g}_{\|}^{\prime},-\boldsymbol{k}_{\|}-\boldsymbol{g}_{\|} ; z^{\prime}, z ; \omega\right) \\
& \times \widetilde{j}_{m}\left(-\boldsymbol{k}_{\|}-\boldsymbol{g}_{\|}, z, \omega\right) d z
\end{aligned}
$$

we obtain

$$
\begin{aligned}
G_{l m}(- & \left.\boldsymbol{k}_{\|}-\boldsymbol{g}_{\|}^{\prime},-\boldsymbol{k}_{\|}-\boldsymbol{g}_{\|} ; z^{\prime}, z ; \omega\right) \\
= & -\frac{\omega \mu_{0}}{2 k_{z, v}\left(\boldsymbol{k}_{\|}+\boldsymbol{g}_{\|}, \omega\right)} \\
& \times \sum_{\alpha} M_{l m}^{\alpha-}\left(-\boldsymbol{k}_{\|}-\boldsymbol{g}_{\|}^{\prime},-\boldsymbol{k}_{\|}-\boldsymbol{g}_{\|} ; z^{\prime}, z ; \omega\right),
\end{aligned}
$$

where $M_{l m}^{\alpha-}$ is defined by

$$
\begin{aligned}
& M_{l m}^{\alpha-}\left(-\boldsymbol{k}_{\|}-\boldsymbol{g}_{\|}^{\prime},-\boldsymbol{k}_{\|}-\boldsymbol{g}_{\|} ; z^{\prime}, z ; \omega\right) \\
& \quad \equiv \sum_{n} M_{l n}\left(-\boldsymbol{k}_{\|}-\boldsymbol{g}_{\|}^{\prime},-\boldsymbol{k}_{\|}-\boldsymbol{g}_{\|} ; z^{\prime}, z ; \omega\right) \hat{\alpha}_{-\boldsymbol{k}_{\|}-\boldsymbol{g}_{\|}, n} \hat{\alpha}_{-\boldsymbol{k}_{\|}-\boldsymbol{g}_{\|}, m} .
\end{aligned}
$$

$\stackrel{\leftrightarrow}{M}^{\alpha-}\left(-\boldsymbol{k}_{\|}-\boldsymbol{g}_{\|}^{\prime},-\boldsymbol{k}_{\|}-\boldsymbol{g}_{\|} ; z^{\prime}, z ; \omega\right)$ can be viewed as the electric field of the parallel wave vector $-\boldsymbol{k}_{\|}-\boldsymbol{g}_{\|}^{\prime}$ at $z=z^{\prime}$ resulting from the $\alpha$-polarized electric current of unit amplitude and the parallel wave vector $-\boldsymbol{k}_{\|}-\boldsymbol{g}_{\|}$at $z=z\left(>z^{\prime}\right)$ and frequency $\omega$. Substituting Eq. (21) into Eq. (15), we obtain

$$
\begin{aligned}
G_{l m}\left(\boldsymbol{r}_{\|}, \boldsymbol{r}_{\|}^{\prime} ; z, z^{\prime} ; \omega\right)= & \sum_{\alpha} \sum_{\boldsymbol{k}_{\|}} \sum_{\boldsymbol{g}_{\|, \boldsymbol{g}_{\|}^{\prime}}} \frac{i}{2 k_{z, v}\left(\boldsymbol{k}_{\|}+\boldsymbol{g}_{\|}, \omega\right) \int d \boldsymbol{r}_{\|}} \\
& \times M_{m l}^{\alpha-}\left(-\boldsymbol{k}_{\|}-\boldsymbol{g}_{\|}^{\prime},-\boldsymbol{k}_{\|}-\boldsymbol{g}_{\|} ; z^{\prime}, z ; \omega\right) \\
& \times e^{i\left(\boldsymbol{k}_{\|}+\boldsymbol{g}_{\|}\right) \cdot \boldsymbol{r}_{\|}-i\left(\boldsymbol{k}_{\|}+\boldsymbol{g}_{\|}^{\prime}\right) \cdot \boldsymbol{r}_{\|}^{\prime}}
\end{aligned}
$$

Hence, we can find the real-space Green's dyadic from the transfer matrix using Eq. (23). The Green's dyadic will now be used to derive Kirchhoff's law (Sec. III) and to calculate the field correlations (Sec. IV).

\section{KIRCHHOFF'S LAW FOR PHOTONIC CRYSTALS}

Kirchhoff's law is derived with the assumption that both (1) the length of the object and (2) the distance from the object is much larger than the wavelength. The first condition is imposed in order to use geometric optics and the second to exclude near fields where evanescent waves exist. As the two assumptions break down, interesting phenomena that do not necessarily obey Kirchhoff's law can be expected. Here, we modify the first assumption by considering periodic struc- tures whose structural components are comparable to or less than the wavelength in size but whose overall dimension is much larger than the wavelength. The second assumption of the far field is still imposed. In this case, Kirchhoff's law is obeyed as will be shown below.

A Fourier component of the electric field in vacuum above the structure $(z>0)$ can be expressed in terms of the field at the surface $(z=0)$ by

$$
\widetilde{\boldsymbol{E}}\left(\boldsymbol{k}_{\|}+\boldsymbol{g}_{\|}, z, \omega\right)=\widetilde{\boldsymbol{E}}\left(\boldsymbol{k}_{\|}+\boldsymbol{g}_{\|}, 0, \omega\right) e^{i k_{z, v}\left(\boldsymbol{k}_{\|}+\boldsymbol{g}_{\|,}, \omega\right) z} .
$$

Substituting Eq. (24) into Eq. (6) with $\boldsymbol{V}=\boldsymbol{E}$ and using the method of stationary phase, ${ }^{22}$ we can obtain the far field form of the electric field as

$$
\boldsymbol{E}\left(\boldsymbol{r}_{\|}, z, \omega\right) \rightarrow-\frac{S}{2 \pi} i \frac{\omega}{c} \cos \theta \frac{e^{i \omega r / c}}{r} \tilde{\boldsymbol{E}}(\boldsymbol{\kappa}, 0, \omega),
$$

where $r=\left|\boldsymbol{r}_{\|}+z \hat{z}\right|, \quad \boldsymbol{\kappa}=\frac{\omega}{c} \frac{\boldsymbol{r}_{\|}}{r}$ is the wave vector parallel to the film surface, and $\theta$ is the angle between the propagation direction and the $z$ axis. Note that $\boldsymbol{\kappa}$ is for the propagating wave and its magnitude is always less than or equal to $\omega / c$

To take the polarization into account, we define the polarization-dependent Green's dyadic $\stackrel{\leftrightarrow}{G}^{\alpha \pm}(\alpha=s$ or $p)$ as

$$
\stackrel{\leftrightarrow}{G}^{\alpha+}\left(\boldsymbol{\kappa}, \boldsymbol{k}_{\|}+\boldsymbol{g}_{\|}^{\prime} ; 0, z^{\prime} ; \omega\right) \equiv \hat{\boldsymbol{\alpha}} \hat{\boldsymbol{\alpha}} \cdot \stackrel{\leftrightarrow}{G}\left(\boldsymbol{\kappa}, \boldsymbol{k}_{\|}+\boldsymbol{g}_{\|}^{\prime} ; 0, z^{\prime} ; \omega\right)
$$

and

$$
\stackrel{\leftrightarrow}{G}^{\alpha-}\left(-\boldsymbol{k}_{\|}-\boldsymbol{g}_{\|}^{\prime},-\boldsymbol{\kappa} ; z^{\prime}, 0 ; \omega\right) \equiv \stackrel{\leftrightarrow}{G}\left(-\boldsymbol{k}_{\|}-\boldsymbol{g}_{\|}^{\prime},-\boldsymbol{\kappa} ; z^{\prime}, 0 ; \omega\right) \cdot \hat{\boldsymbol{\alpha}} \hat{\boldsymbol{\alpha}}
$$

where $\hat{\boldsymbol{\alpha}} \hat{\boldsymbol{\alpha}}=\hat{\boldsymbol{\alpha}}_{-\boldsymbol{\kappa},-} \hat{\boldsymbol{\alpha}}_{-\boldsymbol{\kappa},-}=\hat{\boldsymbol{\alpha}}_{\boldsymbol{\kappa},+} \hat{\boldsymbol{\alpha}}_{\boldsymbol{\kappa},+}$ with $\hat{\boldsymbol{\alpha}}=\hat{\boldsymbol{s}}$ or $\hat{\boldsymbol{p}}$ and using the same notation as in Eq. (16). The Fourier component of the electric field of the $\alpha$-polarized wave going in the $\pm z$ direction at $z=0$ is obtained as

$$
\widetilde{\boldsymbol{E}}^{\alpha \pm}(\boldsymbol{\kappa}, 0, \omega)=\hat{\boldsymbol{\alpha}} \hat{\boldsymbol{\alpha}} \cdot \widetilde{\boldsymbol{E}}(\boldsymbol{\kappa}, 0, \omega) .
$$

From Eqs. (7), (13), (26), and (27) letting $\boldsymbol{k}_{\|}+\boldsymbol{g}_{\|}=\boldsymbol{\kappa}$, we obtain $\widetilde{\boldsymbol{E}}^{\alpha+}$ using the inverse Fourier transform of $\tilde{\boldsymbol{j}}$ as

$$
\begin{aligned}
\widetilde{E}_{l}^{\alpha+}(\boldsymbol{\kappa}, 0, \omega)= & \frac{1}{S} \sum_{\boldsymbol{g}_{\|}^{\prime}} \int d \boldsymbol{r}_{\|}^{\prime} \int_{-L}^{0} d z^{\prime} e^{-i\left(\boldsymbol{k}_{\|}+\boldsymbol{g}_{\|}^{\prime}\right) \cdot \boldsymbol{r}_{\|}^{\prime}} \\
& \times G_{l m}^{\alpha+}\left(\boldsymbol{\kappa}, \boldsymbol{k}_{\|}+\boldsymbol{g}_{\|}^{\prime} ; 0, z^{\prime} ; \omega\right) j_{m}\left(\boldsymbol{r}_{\|}^{\prime}, z^{\prime}, \omega\right) .
\end{aligned}
$$

The ensemble average of $\widetilde{\boldsymbol{E}}^{\alpha+*}(\boldsymbol{\kappa}, 0, \omega) \cdot \widetilde{\boldsymbol{E}}^{\alpha+}\left(\boldsymbol{\kappa}, 0, \omega^{\prime}\right)$ is obtained from Eq. (28) as

$$
\begin{aligned}
\left\langle\widetilde{\boldsymbol{E}}^{\alpha+*}\right. & \left.(\boldsymbol{\kappa}, 0, \omega) \cdot \widetilde{\boldsymbol{E}}^{\alpha+}\left(\boldsymbol{\kappa}, 0, \omega^{\prime}\right)\right\rangle \\
= & \frac{\omega}{\pi S} \varepsilon_{0} \delta\left(\omega-\omega^{\prime}\right) \sum_{l, m} \sum_{\boldsymbol{g}_{\|}^{\prime} \boldsymbol{g}_{\|}^{\prime \prime}} \int_{-L}^{0} d z^{\prime} \widetilde{f}\left(\boldsymbol{g}_{\|}^{\prime \prime}-\boldsymbol{g}_{\|}^{\prime}, z^{\prime}, \omega\right) \\
& \times G_{l m}^{\alpha+*}\left(\boldsymbol{\kappa}, \boldsymbol{k}_{\|}+\boldsymbol{g}_{\|}^{\prime} ; 0, z^{\prime} ; \omega\right) G_{l m}^{\alpha+}\left(\boldsymbol{\kappa}, \boldsymbol{k}_{\|}+\boldsymbol{g}_{\|}^{\prime \prime} ; 0, z^{\prime} ; \omega\right),
\end{aligned}
$$

where $\varepsilon_{0}$ is the permittivity of free space and 


$$
\begin{aligned}
\widetilde{f}\left(\boldsymbol{g}_{\|}^{\prime \prime}-\boldsymbol{g}_{\|}^{\prime}, z^{\prime}, \omega\right)= & \frac{1}{S} \int d \boldsymbol{r}_{\|}^{\prime} e^{i\left(\boldsymbol{g}_{\|}^{\prime}-\boldsymbol{g}_{\|}^{\prime \prime}\right) \cdot \boldsymbol{r}_{\|}^{\prime}} \varepsilon_{I}\left(\mathbf{r}_{\|}^{\prime}, z^{\prime}, \omega\right) \\
& \times \frac{\hbar \omega}{e^{\hbar \omega / k T\left(\mathbf{r}_{\|}^{\prime}, z^{\prime}\right)}-1}
\end{aligned}
$$

$\tilde{f}$ is the Fourier transform of a function $f\left(\boldsymbol{r}_{\|}^{\prime}, z^{\prime}, \omega\right)$ $\equiv \varepsilon_{I}\left(\boldsymbol{r}_{\|}^{\prime}, z^{\prime}, \omega\right) \Theta\left[\omega, T\left(\boldsymbol{r}_{\|}^{\prime}, z^{\prime}\right)\right]$. Here $f$ is assumed to be periodic in the $x y$ plane. In Eq. (30), the two integrations can be done for a unit cell without changing the results, so that the calculation of $\tilde{f}$ becomes simple. Note that the integration is evaluated only over the material portion because $\varepsilon_{I}=0$ in vacuum. In deriving Eq. (29), we used the fluctuationdissipation theorem assuming local equilibrium ${ }^{17}$

$$
\begin{aligned}
& \left\langle j_{l}^{*}\left(\boldsymbol{r}_{\|}^{\prime}, z^{\prime}, \omega, T\right) j_{m}\left(\boldsymbol{r}_{\|}^{\prime \prime}, z^{\prime \prime}, \omega^{\prime}, T\right)\right\rangle \\
& =\frac{\omega \Theta(\omega, T)}{\pi} \varepsilon_{0} \varepsilon_{I}\left(\boldsymbol{r}_{\|}^{\prime}, z^{\prime}, \omega\right) \delta_{l m} \delta\left(\boldsymbol{r}_{\|}^{\prime}-\boldsymbol{r}_{\|}^{\prime \prime}\right) \delta\left(z^{\prime}-z^{\prime \prime}\right) \\
& \quad \times \delta\left(\omega-\omega^{\prime}\right),
\end{aligned}
$$

where $\varepsilon_{I}$ is the imaginary part of the dielectric function and $\Theta(\omega, T)=\frac{\hbar \omega}{e^{\hbar \omega / k_{B} T}-1}$ is the mean energy of the harmonic oscillator minus the zero-point energy. Equation (31) says that the thermal currents are delta correlated in position. This is the consequence of the assumption that the medium is without spatial dispersion.

The magnitude of the ensemble-averaged Poynting vector $\left\langle S_{\omega}^{\alpha+}\left(\boldsymbol{r}_{\|}, z\right)\right\rangle$ of light of $\alpha$ polarization and frequency $\omega$ propagating in the $+z$ direction is given by ${ }^{23}$

$$
\left\langle S_{\omega}^{\alpha+}\left(\boldsymbol{r}_{\|}, z\right)\right\rangle \delta\left(\omega-\omega^{\prime}\right)=\frac{2}{\mu_{0} c}\left\langle\boldsymbol{E}^{\alpha+*}\left(\boldsymbol{r}_{\|}, z, \omega\right) \cdot \boldsymbol{E}^{\alpha+}\left(\boldsymbol{r}_{\|}, z, \omega^{\prime}\right)\right\rangle .
$$

The emission rate of $\alpha$-polarized light per unit solid angle per unit frequency per unit area in the far field is

$$
\mathcal{P}^{\alpha+}(\boldsymbol{\kappa}, \omega)=\frac{r^{2}}{S \cos \theta}\left\langle S_{\omega}^{\alpha+}\left(\boldsymbol{r}_{\|}, z\right)\right\rangle_{\omega r / c \rightarrow \infty} .
$$

We find the Poynting vector from Eqs. (25), (29), and (32) and insert this into Eq. (33) to obtain the emission rate. Then the resulting expression is

$$
\begin{aligned}
\mathcal{P}^{\alpha+}(\boldsymbol{\kappa}, \omega)= & \frac{\omega^{3} \cos \theta}{2 \pi^{3} c^{3}} \frac{\varepsilon_{0}}{\mu_{0}} \sum_{l, m} \sum_{\boldsymbol{g}_{\|}^{\prime}, \boldsymbol{g}_{\|}^{\prime \prime}} \int_{-L}^{0} d z^{\prime} \tilde{f}\left(\boldsymbol{g}_{\|}^{\prime \prime}-\boldsymbol{g}_{\|}^{\prime}, z^{\prime}, \omega\right) \\
& \times G_{l m}^{\alpha+*}\left(\boldsymbol{\kappa}, \boldsymbol{k}_{\|}+\boldsymbol{g}_{\|}^{\prime} ; 0, z^{\prime} ; \omega\right) G_{l m}^{\alpha+}\left(\boldsymbol{\kappa}, \boldsymbol{k}_{\|}+\boldsymbol{g}_{\|}^{\prime \prime} ; 0, z^{\prime} ; \omega\right) .
\end{aligned}
$$

Now, as in Kirchhoff's law, we wish to relate the emission rate to the parameters in the absorption process. For this, we use the electrodynamic reciprocity theorem Eq. (14) for $\boldsymbol{k}_{\|}+\boldsymbol{g}_{\|}=\boldsymbol{\kappa}$ and $z=0$. We premultiply $\hat{\boldsymbol{\alpha}} \hat{\boldsymbol{\alpha}}$ with Eq. (14) and use Eq. (26) to find the relation for polarization $\alpha$ as

$$
\stackrel{\leftrightarrow}{G}^{\alpha+}\left(\boldsymbol{\kappa}, \boldsymbol{k}_{\|}+\boldsymbol{g}_{\|}^{\prime} ; 0, z^{\prime} ; \omega\right)=\stackrel{\leftrightarrow}{G}^{\alpha-}{ }^{T}\left(-\boldsymbol{k}_{\|}-\boldsymbol{g}_{\|}^{\prime},-\boldsymbol{\kappa} ; z^{\prime}, 0 ; \omega\right)
$$

Substituting Eq. (35) into Eq. (34), we have

$$
\begin{aligned}
\mathcal{P}^{\alpha+}(\boldsymbol{\kappa}, \omega)= & \frac{\omega^{3} \cos \theta}{2 \pi^{3} c^{3}} \frac{\varepsilon_{0}}{\mu_{0}} \sum_{l, m} \sum_{\boldsymbol{g}_{\|}^{\prime}, \boldsymbol{g}_{\|}^{\prime \prime}} \int_{-L}^{0} d z^{\prime} \tilde{f}\left(\boldsymbol{g}_{\|}^{\prime \prime}-\boldsymbol{g}_{\|}^{\prime}, z^{\prime}, \omega\right) \\
& \times G_{l m}^{\alpha-*}\left(-\boldsymbol{k}_{\|}-\boldsymbol{g}_{\|}^{\prime},-\boldsymbol{\kappa} ; z^{\prime}, 0 ; \omega\right) \\
& \times G_{l m}^{\alpha-}\left(-\boldsymbol{k}_{\|}-\boldsymbol{g}_{\|}^{\prime \prime},-\boldsymbol{\kappa} ; z^{\prime}, 0 ; \omega\right) .
\end{aligned}
$$

In calculating this emission rate, we may use the Green's dyadic in Eq. (21). Postmultiplying $\hat{\boldsymbol{\alpha}} \hat{\boldsymbol{\alpha}}$ with Eq. (21) for $\boldsymbol{k}_{\|}+\boldsymbol{g}_{\|}=\boldsymbol{\kappa}$ and $z=0$, and using Eq. (26) and the orthogonality for different polarizations defined in Eq. (16), we obtain the relation

$$
\begin{aligned}
\stackrel{\leftrightarrow}{G}^{\alpha-} & \left(-\boldsymbol{k}_{\|}-\boldsymbol{g}_{\|}^{\prime},-\boldsymbol{\kappa} ; z^{\prime}, 0 ; \omega\right) \\
& =-\frac{c \mu_{0}}{2 \cos \theta} \stackrel{\leftrightarrow}{M}^{\alpha-}\left(-\boldsymbol{k}_{\|}-\boldsymbol{g}_{\|}^{\prime},-\boldsymbol{\kappa} ; z^{\prime}, 0 ; \omega\right),
\end{aligned}
$$

where we used $k_{z, v}(\boldsymbol{\kappa}, \omega)=\frac{\omega}{c} \cos \theta$. Plugging Eq. (37) into Eq. (36), we find that

$$
\begin{aligned}
\mathcal{P}^{\alpha+}(\boldsymbol{\kappa}, \omega)= & \frac{\omega^{3}}{8 \pi^{3} c^{3} \cos \theta} \sum_{l, m} \sum_{\boldsymbol{g}_{\|}^{\prime}, \boldsymbol{g}_{\|}^{\prime \prime}} \int_{-L}^{0} d z^{\prime} \tilde{f}\left(\boldsymbol{g}_{\|}^{\prime \prime}-\boldsymbol{g}_{\|}^{\prime}, z^{\prime}, \omega\right) \\
& \times M_{l m}^{\alpha-*}\left(-\boldsymbol{k}_{\|}-\boldsymbol{g}_{\|}^{\prime},-\boldsymbol{\kappa} ; z^{\prime}, 0 ; \omega\right) \\
& \times M_{l m}^{\alpha-}\left(-\boldsymbol{k}_{\|}-\boldsymbol{g}_{\|}^{\prime \prime},-\boldsymbol{\kappa} ; z^{\prime}, 0 ; \omega\right) .
\end{aligned}
$$

Hence the emission rate can be calculated using the transfer matrices.

By expressing transfer matrices in terms of the electric fields, the physics becomes clearer. Similarly to Eq. (19), the electric field at a plane $z=z^{\prime}<0$ with parallel wave vector $-\boldsymbol{k}_{\|}-\boldsymbol{g}_{\|}^{\prime}$ resulting from the incident wave of polarization $\alpha$ and parallel wave vector $-\boldsymbol{\kappa}$ is

$$
\begin{aligned}
& d \widetilde{\boldsymbol{E}}_{(-\boldsymbol{\kappa}, 0)}^{(\alpha-)}\left(-\boldsymbol{k}_{\|}-\boldsymbol{g}_{\|}^{\prime}, z^{\prime}, \omega\right) \\
& \quad=\stackrel{\leftrightarrow}{M}^{\alpha-}\left(-\boldsymbol{k}_{\|}-\boldsymbol{g}_{\|}^{\prime},-\boldsymbol{\kappa} ; z^{\prime}, 0 ; \omega\right) \cdot d \widetilde{\boldsymbol{E}}_{i n c}^{\alpha-}(-\boldsymbol{\kappa}, 0, \omega) .
\end{aligned}
$$

In Eq. (39) and the following, the brackets in the subscript or superscript are used to describe the incident light that leads to a quantity inside the film, in this case $d \widetilde{\boldsymbol{E}}$. The subscripts indicate the parallel wave vector of the incident light on the film at $z=0$. The superscripts indicate the polarization and the propagation direction of the incident light. For example, the electric field $\widetilde{\boldsymbol{E}}_{(-\boldsymbol{\kappa}, 0)}^{(\alpha)}$ in Eq. (39) results from the incident light that is $\alpha$ polarized, propagating downward, and having parallel wave vector $\boldsymbol{- \kappa}$. Using Eq. (39), we obtain the transfer matrix as

$$
\begin{aligned}
\stackrel{M}{M}^{\alpha-}\left(-\boldsymbol{k}_{\|}-\boldsymbol{g}_{\|}^{\prime},-\boldsymbol{\kappa} ; z^{\prime}, 0 ; \omega\right) \\
=\frac{\widetilde{\boldsymbol{E}}_{(-\boldsymbol{\kappa}, 0)}^{(\alpha-)}\left(-\boldsymbol{k}_{\|}-\boldsymbol{g}_{\|}^{\prime}, z^{\prime}, \omega\right) \widetilde{\boldsymbol{E}}_{i n c}^{\alpha-}(-\boldsymbol{\kappa}, 0, \omega)}{\left[\widetilde{\boldsymbol{E}}_{i n c}^{\alpha-}(-\boldsymbol{\kappa}, 0, \omega)\right]^{2}} .
\end{aligned}
$$

Inserting Eqs. (30) and (40) into Eq. (38) and using [see Eq. (3)] 


$$
\boldsymbol{E}_{-\boldsymbol{k}_{\|}(-\boldsymbol{\kappa}, 0)}^{(\alpha-)}\left(\boldsymbol{r}_{\|}^{\prime}, z^{\prime}, \omega\right)=\sum_{\boldsymbol{g}_{\|}^{\prime}} \widetilde{\boldsymbol{E}}_{(-\boldsymbol{\kappa}, 0)}^{(\alpha-)}\left(-\boldsymbol{k}_{\|}-\boldsymbol{g}_{\|}^{\prime}, z^{\prime}, \omega\right) e^{-i\left(\boldsymbol{k}_{\|}+\boldsymbol{g}_{\|}^{\prime}\right) \cdot \boldsymbol{r}_{\|}^{\prime}},
$$

we obtain

$$
\begin{aligned}
\mathcal{P}^{\alpha+}(\boldsymbol{\kappa}, \omega)= & \frac{\hbar \omega^{4}}{8 \pi^{3} c^{3} S \cos \theta} \int_{-L}^{0} d z^{\prime} \int d \boldsymbol{r}_{\|}^{\prime} \\
& \times \frac{\varepsilon_{I}\left(\boldsymbol{r}_{\|}^{\prime}, z^{\prime}, \omega\right)}{e^{\hbar \omega / k T\left(\boldsymbol{r}_{\|}^{\prime}, z^{\prime}\right)}-1} \frac{\left|\boldsymbol{E}_{-\boldsymbol{k}_{\|}(-\boldsymbol{\kappa}, 0)}^{(\alpha-)}\left(\boldsymbol{r}_{\|}^{\prime}, z^{\prime}, \omega\right)\right|^{2}}{\left|\widetilde{\boldsymbol{E}}^{\alpha-}(-\boldsymbol{\kappa}, 0, \omega)\right|^{2}},
\end{aligned}
$$

where we suppressed the subscript inc on $\widetilde{\boldsymbol{E}}^{\alpha-}$. In Eqs. (41) and (42), the electric field $\boldsymbol{E}_{-\boldsymbol{k}_{\|}(-\boldsymbol{\kappa}, 0)}^{(\alpha-)}$ has a parallel wave vector $-\boldsymbol{k}_{\|}$in the first Brillouin zone and results from $\alpha$-polarized light with parallel wave vector $-\boldsymbol{\kappa}$ incident on the $z=0$ plane. Because we assumed that the temperature distribution has the same periodicity as the structure, the integrations in Eq. (42) can be performed over a surface unit cell. Then Eq. (42) can be written as

$$
\begin{aligned}
\mathcal{P}^{\alpha+}(\boldsymbol{\kappa}, \omega)= & \frac{1}{S_{u c} \cos \theta} \int_{-L}^{0} d z^{\prime} \int_{u c} d \boldsymbol{r}_{\|}^{\prime} \times Q_{(-\boldsymbol{\kappa}, 0)}^{(\alpha-)} \\
& \times\left(\boldsymbol{r}_{\|}^{\prime}, z^{\prime}, \omega\right) \mathcal{P}_{B B}^{\alpha+}\left(\boldsymbol{r}_{\|}^{\prime}, z^{\prime}, \omega\right),
\end{aligned}
$$

where $Q_{(-\boldsymbol{\kappa}, 0)}^{(\alpha-)}$ is the local absorption rate per unit volume when the film is irradiated with $\alpha$-polarized light of unit intensity with parallel wave vector $-\boldsymbol{\kappa}$ incident on the $z=0$ plane. This absorption rate is given by ${ }^{20}$

$$
Q_{(-\boldsymbol{\kappa}, 0)}^{(\alpha-)}\left(\boldsymbol{r}_{\|}^{\prime}, z^{\prime}, \omega\right)=\frac{\omega}{c} \varepsilon_{I}\left(\boldsymbol{r}_{\|}^{\prime}, z^{\prime}, \omega\right) \frac{\left|\boldsymbol{E}_{-k_{\|}(-\boldsymbol{\kappa}, 0)}^{(\alpha-)}\left(\boldsymbol{r}_{\|}^{\prime}, z^{\prime}, \omega\right)\right|^{2}}{\left|\widetilde{\boldsymbol{E}}^{\alpha-}(-\boldsymbol{\kappa}, 0, \omega)\right|^{2}} .
$$

Finally, $\mathcal{P}_{B B}^{\alpha+}$ is the black-body radiation intensity for $\alpha$-polarized light given by

$$
\mathcal{P}_{B B}^{\alpha+}\left(\boldsymbol{r}_{\|}^{\prime}, z^{\prime}, \omega\right)=\frac{\omega^{2}}{8 \pi^{3} c^{2}} \frac{\hbar \omega}{e^{\hbar \omega / k T\left(r_{\|}^{\prime}, z^{\prime}\right)}-1} .
$$

Equation (43) is the same as the Eq. (1) of Ref. 24 except for slight changes in notation. When the temperature is uniform, $\mathcal{P}_{B B}^{\alpha+}$ is independent of the position and Eq. (43) becomes

$$
\mathcal{P}^{\alpha+}(\boldsymbol{\kappa}, \omega)=A^{(\alpha-)}(-\boldsymbol{\kappa}, \omega) \mathcal{P}_{B B}^{\alpha+}(\omega),
$$

where $A^{(\alpha-)}$ is the absorptivity for an $\alpha$-polarized incident wave given by

$$
A^{(\alpha-)}(-\boldsymbol{\kappa}, \omega)=\frac{1}{S_{u c} \cos \theta} \int_{-L}^{0} d z^{\prime} \int_{u c} d \boldsymbol{r}_{\|}^{\prime} Q_{(-\kappa, 0)}^{(\alpha-)}\left(\boldsymbol{r}_{\|}^{\prime}, z^{\prime}, \omega\right)
$$

Equation (46) is Kirchhoff's law for a periodically structured object with an arbitrary length scale. Thus, we proved that the Kirchhoff's law is obeyed for photonic crystal films if local equilibrium is assumed.

\section{OPTICAL COHERENCE FOR PHOTONIC CRYSTALS}

The correlation between the electric fields of a given frequency at two different points is represented by the electric cross-spectral density tensor $W_{l m}^{(e)}$, defined by ${ }^{22}$

$$
\left\langle E_{l}^{*}\left(\boldsymbol{r}_{1}, \omega\right) E_{m}\left(\boldsymbol{r}_{2}, \omega^{\prime}\right)\right\rangle=W_{l m}^{(e)}\left(\boldsymbol{r}_{1}, \boldsymbol{r}_{2}, \omega\right) \delta\left(\omega-\omega^{\prime}\right) .
$$

This can be obtained from Eqs. (8), (31), and (48) as

$$
\begin{aligned}
W_{l m}^{(e)}\left(\boldsymbol{r}_{\| 1}, \boldsymbol{r}_{\| 2} ; z_{1}, z_{2} ; \omega\right)= & \frac{\omega^{3} \mu_{0}^{2} \varepsilon_{0}}{\pi} \int d \boldsymbol{r}_{\|}^{\prime} \int d z^{\prime} \\
& \times \varepsilon_{I}\left(\boldsymbol{r}_{\|}^{\prime}, z^{\prime}, \omega\right) \Theta\left[\omega, T\left(\boldsymbol{r}_{\|}^{\prime}, z^{\prime}\right)\right] \\
& \times \sum_{n} G_{l n}^{*}\left(\boldsymbol{r}_{\| 1}, \boldsymbol{r}_{\|}^{\prime} ; z_{1}, z^{\prime} ; \omega\right) \\
& \times G_{m n}\left(\boldsymbol{r}_{\| 2}, \boldsymbol{r}_{\|}^{\prime} ; z_{2}, z^{\prime} ; \omega\right) .
\end{aligned}
$$

In Eq. (49), we assumed local equilibrium for nonuniform temperatures because the fluctuation-dissipation theorem was used. This is in general justified for high-vacuum conditions. ${ }^{24}$ The calculation of $W_{l m}^{(e)}$ for an arbitrary temperature distribution is formidable because of the integration in Eq. (49). However, if $f\left(\boldsymbol{r}_{\|}^{\prime}, z^{\prime}, \omega\right)$ $\equiv \varepsilon_{I}\left(\boldsymbol{r}_{\|}^{\prime}, z^{\prime}, \omega\right) \Theta\left[\omega, T\left(\boldsymbol{r}_{\|}^{\prime}, z^{\prime}\right)\right]$ is periodic in the $x y$ plane, the calculation is simplified using Eq. (30). In this case, $f$ can be expanded in a Fourier series as

$$
f\left(\boldsymbol{r}_{\|}^{\prime}, z^{\prime}, \omega\right)=\sum_{g_{\|}^{\prime}} \tilde{f}\left(\boldsymbol{g}_{\|}^{\prime}, z^{\prime}\right) e^{i \boldsymbol{g}_{\|}^{\prime} \cdot \boldsymbol{r}_{\|}^{\prime}} .
$$

Substituting Eq. (50) into Eq. (49) and using Eq. (23), we obtain

$$
\begin{aligned}
W_{l m}^{(e)}\left(\boldsymbol{r}_{\| 1}, \boldsymbol{r}_{\| 2} ; z_{1}, z_{2} ; \omega\right) & \\
= & \frac{\omega^{3} \mu_{0}^{2} \varepsilon_{0}}{\pi S} \int d z^{\prime} \sum_{n} \sum_{\alpha, \beta} \sum_{\boldsymbol{k}_{\|}} \sum_{\boldsymbol{g}_{\| 1}, \boldsymbol{g}_{\| 1}^{\prime}} \sum_{\boldsymbol{g}_{\| 2}, \boldsymbol{g}_{\| 2}^{\prime}} \tilde{f}\left(\boldsymbol{g}_{\| 2}^{\prime}-\boldsymbol{g}_{\| 1}^{\prime}, z^{\prime}\right) \\
& \times \frac{e^{-i\left(\boldsymbol{k}_{\|}+\boldsymbol{g}_{\| 1}\right) \cdot \boldsymbol{r}_{\| 1}+i\left(\boldsymbol{k}_{\|}+\boldsymbol{g}_{\| 2}\right) \cdot \boldsymbol{r}_{\| 2}}}{4 k_{z, v}^{*}\left(\boldsymbol{k}_{\|}+\boldsymbol{g}_{\| 1}, \omega\right) k_{z, v}\left(\boldsymbol{k}_{\|}+\boldsymbol{g}_{\| 2}, \omega\right)} \\
& \times e^{-i k_{z, v}^{*}\left(\boldsymbol{k}_{\|}+\boldsymbol{g}_{\| 1}, \omega\right) z_{1}+i k_{z, v}\left(\boldsymbol{k}_{\|}+\boldsymbol{g}_{\| 2}, \omega\right) z_{2}} \\
& \times M_{n l}^{\alpha-*}\left(-\boldsymbol{k}_{\|}-\boldsymbol{g}_{\| 1}^{\prime},-\boldsymbol{k}_{\|}-\boldsymbol{g}_{\| 1} ; z^{\prime}, 0 ; \omega\right) \\
& \times M_{n m}^{\beta-}\left(-\boldsymbol{k}_{\|}-\boldsymbol{g}_{\| 2}^{\prime},-\boldsymbol{k}_{\|}-\boldsymbol{g}_{\| 2} ; z^{\prime}, 0 ; \omega\right),
\end{aligned}
$$

where $\beta$ is the polarization defined similarly to $\alpha$ and we used

$$
\begin{aligned}
\stackrel{\leftrightarrow}{M} & \left.-\boldsymbol{k}_{\|}-\boldsymbol{g}_{\|}^{\prime},-\boldsymbol{k}_{\|}-\boldsymbol{g}_{\|} ; z^{\prime}, z ; \omega\right) \\
& =\overleftrightarrow{M}\left(-\boldsymbol{k}_{\|}-\boldsymbol{g}_{\|}^{\prime},-\boldsymbol{k}_{\|}-\boldsymbol{g}_{\|} ; z^{\prime}, 0 ; \omega\right) e^{i k_{z, v}\left(\boldsymbol{k}_{\|}+\boldsymbol{g}_{\|,}, \omega\right) z}
\end{aligned}
$$

and

$$
\sum_{\boldsymbol{g}_{\|}^{\prime}} \tilde{f}\left(\boldsymbol{g}_{\|}^{\prime}, z^{\prime}\right) \delta_{\boldsymbol{g}_{\|}^{\prime}+\left(\boldsymbol{k}_{\| 1}+\boldsymbol{g}_{\| 1}^{\prime}\right)-\left(\boldsymbol{k}_{\| 2}+\boldsymbol{g}_{\| 2}^{\prime}\right), 0}=\tilde{f}\left(\boldsymbol{g}_{\| 2}^{\prime}-\boldsymbol{g}_{\| 1}^{\prime}, z^{\prime}\right) \delta_{k_{\| 1}, \boldsymbol{k}_{\| 2}} .
$$

Thus the electric cross-spectral density tensor can be calculated from the knowledge of the transfer matrices using Eq. (51). 
Now we wish to replace the transfer matrices by the electric fields as in the previous section. We replace $\boldsymbol{\kappa}$ in Eqs. (40) and (41) by $\boldsymbol{k}_{\|}+\boldsymbol{g}_{\| 1}$ and $\boldsymbol{k}_{\|}+\boldsymbol{g}_{\| 2}$ and substitute the resulting equations into Eq. (51) to obtain

$$
\begin{aligned}
& W_{l m}^{(e)}\left(\boldsymbol{r}_{\| 1}, \boldsymbol{r}_{\| 2} ; z_{1}, z_{2} ; \omega\right) \\
& =\frac{\omega^{3} \mu_{0}^{2} \varepsilon_{0}}{\pi S^{2}} \int d \boldsymbol{r}_{\|}^{\prime} \int d z^{\prime} \\
& \times \sum_{n} \sum_{\alpha, \beta} \sum_{\boldsymbol{k}_{\|}} \sum_{\boldsymbol{g}_{\| 1}, \boldsymbol{g}_{\| 2}} \frac{\varepsilon_{I}\left(\boldsymbol{r}_{\|}^{\prime}, z^{\prime}, \omega\right) \Theta\left[\omega, T\left(\boldsymbol{r}_{\|}^{\prime}, z^{\prime}\right)\right]}{4 k_{z, v}^{*}\left(\boldsymbol{k}_{\|}+\boldsymbol{g}_{\| 1}, \omega\right) k_{z, v}\left(\boldsymbol{k}_{\|}+\boldsymbol{g}_{\| 2}, \omega\right)} \\
& \times e^{-i\left(\boldsymbol{k}_{\|}+\boldsymbol{g}_{\| 1}\right) \cdot \boldsymbol{r}_{\| 1}+i\left(\boldsymbol{k}_{\|}+\boldsymbol{g}_{\| 2}\right) \cdot \boldsymbol{r}_{\| 2}} e^{-i k_{z, v}^{*}\left(\boldsymbol{k}_{\|}+\boldsymbol{g}_{\| 1}, \omega\right) z_{1}+i k_{z, v}\left(\boldsymbol{k}_{\|}+\boldsymbol{g}_{\| 2}, \omega\right) z_{2}} \\
& \times E_{-\boldsymbol{k}_{\|}\left(-\boldsymbol{k}_{\|}-\boldsymbol{g}_{\| 1}, 0\right), n}^{(\alpha-)}\left(\boldsymbol{r}_{\|}^{\prime}, z^{\prime}, \omega\right) E_{-\boldsymbol{k}_{\|}\left(-\boldsymbol{k}_{\|}-\boldsymbol{g}_{\| 2}, 0\right), n}^{(\beta-)}\left(\boldsymbol{r}_{\|}^{\prime}, z^{\prime}, \omega\right) \\
& \times \frac{\widetilde{E}_{l}^{\alpha-*}\left(-\boldsymbol{k}_{\|}-\boldsymbol{g}_{\| 1}, 0, \omega\right) \tilde{E}_{m}^{\beta-}\left(-\boldsymbol{k}_{\|}-\boldsymbol{g}_{\| 2}, 0, \omega\right)}{\widetilde{\boldsymbol{E}}^{\alpha-* 2}\left(-\boldsymbol{k}_{\|}-\boldsymbol{g}_{\| 1}, 0, \omega\right) \widetilde{\boldsymbol{E}}^{\beta-2}\left(-\boldsymbol{k}_{\|}-\boldsymbol{g}_{\| 2}, 0, \omega\right)},
\end{aligned}
$$

where we removed $\tilde{f}$ using Eq. (30). Equation (54) is the general equation that describes the correlations of any two points over periodic structures with a periodic temperature distribution. The location of the two points can be in the near field where evanescent fields exist.

Often, we are interested in the coherence on a plane, that is, parallel to the surface of the film and in the far field. In this case, the coherence is related to the angular dependence of the thermal emission. ${ }^{22}$ We set $z_{1}=z_{2}=z$ and assume that $z$ is large such that $k_{z I, v} z \gg 1$ for all $\boldsymbol{g}_{\|}$'s, where $k_{z I, v}$ is the imaginary part of $k_{z, v}$. We also assume that the temperature is uniform and the wavelength is larger than the periodicity of the structure. For this wavelength, only zero-order reflections occur for an incident light. With these assumptions, Eq. (54) is reduced to

$$
\begin{aligned}
W_{l m}^{(e)}\left(\boldsymbol{R}_{\|}, z, \omega\right)= & \frac{\omega \mu_{0}}{\pi S S_{u c}} \int_{u c} d \boldsymbol{r}_{\|}^{\prime} \int d z^{\prime} \\
& \times \sum_{n} \sum_{\alpha, \beta} \sum_{\boldsymbol{k}_{\|}} \frac{\varepsilon_{I}\left(\boldsymbol{r}_{\|}^{\prime}, z^{\prime}, \omega\right) \Theta(\omega, T)}{4 \cos ^{2} \theta} e^{i \boldsymbol{k}_{\|} \boldsymbol{R}_{\|}} \\
& \times E_{-\boldsymbol{k}_{\|}\left(-\boldsymbol{k}_{\|}, 0\right), n}^{(\alpha-*}\left(\boldsymbol{r}_{\|}^{\prime}, z^{\prime}, \omega\right) E_{-\boldsymbol{k}_{\|}\left(\boldsymbol{k}_{\|}, 0\right), n}^{\left(\boldsymbol{r}_{\|}^{\prime}, z^{\prime}, \omega\right)} \\
& \times \frac{\widetilde{E}_{l}^{\alpha-*}\left(-\boldsymbol{k}_{\|}, 0, \omega\right) \tilde{E}_{m}^{\beta-}\left(-\boldsymbol{k}_{\|}, 0, \omega\right)}{\widetilde{\boldsymbol{E}}^{\alpha-* 2}\left(-\boldsymbol{k}_{\|}, 0, \omega\right) \widetilde{\boldsymbol{E}}^{\beta-2}\left(-\boldsymbol{k}_{\|}, 0, \omega\right)},
\end{aligned}
$$

where $\boldsymbol{R}_{\|}=\boldsymbol{r}_{\| 2}-\boldsymbol{r}_{\| 1}, \quad\left|\boldsymbol{k}_{\|}\right| \leq \omega / c$, and we used $k_{z, v}\left(-\boldsymbol{k}_{\|}, \omega\right)$ $=\frac{\omega}{c} \cos \theta$. Note that $W_{i j}^{(e)}$ depends only on the difference in the position in this case. The trace of $\stackrel{\leftrightarrow}{W}^{(e)}$ in Eq. (55) becomes

$$
\begin{aligned}
\operatorname{Tr} \stackrel{W}{W}^{(e)}\left(\boldsymbol{R}_{\|}, z, \omega\right)= & \frac{\omega \mu_{0}}{\pi S S_{u c}} \int_{u c} d \boldsymbol{r}_{\|}^{\prime} \int d z^{\prime} \varepsilon_{I}\left(\boldsymbol{r}_{\|}^{\prime}, z^{\prime}, \omega\right) \Theta(\omega, T) \\
& \times \sum_{\boldsymbol{k}_{\|}} \sum_{\alpha} \frac{e^{i \boldsymbol{k}_{\|} \boldsymbol{R}_{\|}}}{4 \cos ^{2} \theta} \frac{\left|\boldsymbol{E}_{-\boldsymbol{k}_{\|}\left(-\boldsymbol{k}_{\|}, 0\right)}^{(\alpha)}\left(\boldsymbol{r}_{\|}^{\prime}, z^{\prime}, \omega\right)\right|^{2}}{\left|\widetilde{\boldsymbol{E}}^{\alpha-}\left(-\boldsymbol{k}_{\|}, 0, \omega\right)\right|^{2}},
\end{aligned}
$$

where we used the fact that $\hat{\boldsymbol{\alpha}}_{-\boldsymbol{k}_{\|,},-}$is real. Using the absorp- tance $A=\Sigma_{\alpha} A^{(\alpha-)} / 2$ defined in Eq. (47), Eq. (56) can be written as

$$
\operatorname{Tr} \stackrel{\leftrightarrow}{W}^{(e)}\left(\boldsymbol{R}_{\|}, z, \omega\right)=\frac{c \mu_{0} \Theta(\omega, T)}{2 \pi S} \sum_{\boldsymbol{k}_{\|}} \frac{A\left(-\boldsymbol{k}_{\|}, \omega\right)}{\cos \theta} e^{i \boldsymbol{k}_{\|} \cdot \boldsymbol{R}_{\|}} .
$$

Equation (57) indicates that, apart from a constant factor, the coherence function $\operatorname{Tr} \stackrel{\leftrightarrow}{W}^{(e)}$ is equal to the Fourier transform of $A / \cos \theta$. Conversely, the absorptance $A$ can be obtained in terms of the coherence function $\operatorname{Tr} \vec{W}^{(e)}$ by the inverse Fourier transform as

$$
A\left(-\boldsymbol{k}_{\|}, \omega\right)=\frac{2 \pi c \cos \theta}{\omega^{2} \mu_{0} \Theta(\omega, T)} \times \int d \boldsymbol{R}_{\|} \operatorname{Tr} \stackrel{\leftrightarrow}{W}^{(e)}\left(\boldsymbol{R}_{\|}, z, \omega\right) e^{-i \boldsymbol{k}_{\|} \cdot \boldsymbol{R}_{\|}} .
$$

An analytical expression for $\operatorname{Tr} \stackrel{\leftrightarrow}{W}^{(e)}$ is obtained for a black body. In Eq. (57), setting $A=1$ and using $\cos \theta$ $=\frac{c}{\omega} k_{z, v}\left(\boldsymbol{k}_{\|}, \omega\right)$ from Eq. (17), we have

$$
\begin{aligned}
& \operatorname{Tr} \stackrel{\leftrightarrow}{W}^{(e)}\left(\boldsymbol{R}_{\|}, z, \omega\right)=\frac{c \mu_{0} \Theta(\omega, T)}{2 \pi S} \sum_{\boldsymbol{k}_{\|}} \frac{e^{i \boldsymbol{k}_{\|} \boldsymbol{R}_{\|}}}{\sqrt{\frac{\omega^{2}}{c^{2}}-\left|\boldsymbol{k}_{\|}\right|^{2}}} \\
& =\frac{c \mu_{0} \Theta(\omega, T)}{8 \pi^{3}} \int_{k_{\|}<\omega / c} d \boldsymbol{k}_{\|} \frac{e^{i \boldsymbol{k}_{\|} \boldsymbol{R}_{\|}}}{\sqrt{\frac{\omega^{2}}{c^{2}}-k_{\|}^{2}}} \\
& =\frac{c \mu_{0} \Theta(\omega, T)}{4 \pi^{2}} \frac{\sin \rho}{\rho},
\end{aligned}
$$

where $\rho=\frac{\omega}{c} R_{\|}$. In Eq. (59), the transition from summation to integral is valid when the linear dimension of the black body is much larger than the wavelength $\lambda=2 \pi c / \omega$. Equation (59) agrees with Ref. 25 and shows that the coherence length of a black body is on the order of $\lambda$. Note that in general $W_{i j}^{(e)}$ in Eq. (55) cannot be obtained in terms of $A^{(\alpha-)}$ because the electric fields resulting from incident $s$ - and $p$-polarized light $\left(\boldsymbol{E}^{(s-)}\right.$ and $\left.\boldsymbol{E}^{(p-)}\right)$ are not necessarily orthogonal in the photonic crystal. This is possible only when they are orthogonal as in a homogeneous flat film. However, $\operatorname{Tr} \stackrel{\leftrightarrow}{W}^{(e)}$ can be expressed in terms of $A$ for photonic crystals as seen in Eq. (57). Equation (57) shows that $\operatorname{Tr} \stackrel{\leftrightarrow}{W}^{(e)}$ is in general a complex number. This can be real if $A\left(-\boldsymbol{k}_{\|}, \omega\right)=A\left(\boldsymbol{k}_{\|}, \omega\right)$ which happens for structures with mirror symmetry. However, even in this case, $\operatorname{Tr} \stackrel{\leftrightarrow}{W}^{(e)}$ in the near field is in general complex.

Using Eq. (57) and the transfer matrix method, ${ }^{26}$ we calculated $\operatorname{Tr} \stackrel{\leftrightarrow}{W}^{(e)}$ for the SiC grating reported in Ref. 27. At $\lambda$ $=11.36 \mu \mathrm{m}$, surface phonon polaritons can be excited on the $\mathrm{SiC}$ film and coupled to light by the grating. Because the surface phonon polaritons can propagate a long distance, it was shown that the coherence length was large. This coherence has already been calculated in Ref. 28 but only the contributions from the surface phonon polaritons were considered. Here we investigate the coherence considering all the propagating fields. The structural parameters were slightly modified such that the groove period, width, and depth are $6.248 \mu \mathrm{m}, 2.083 \mu \mathrm{m}$, and $250 \mathrm{~nm}$, respectively. 


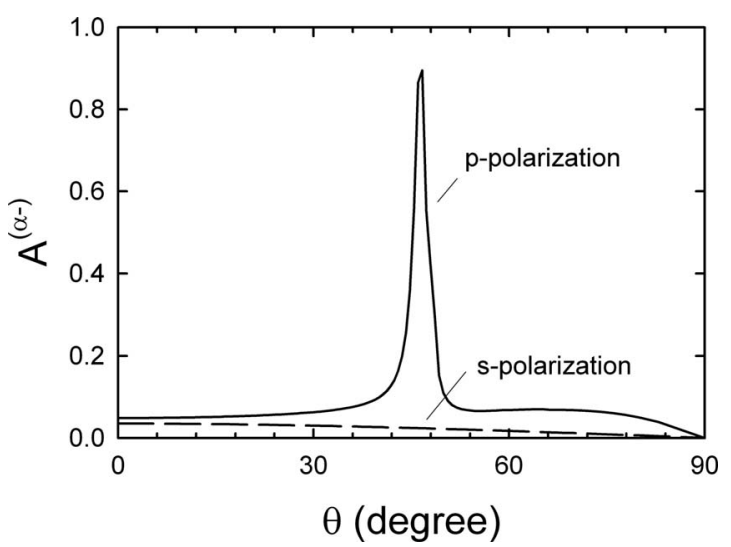

FIG. 1. Angular dependence of the absorptance of a SiC linear grating for $s$ and $p$ polarizations at $\lambda=11.36 \mu \mathrm{m}$. The groove period, width, and depth are $6.248 \mu \mathrm{m}, 2.083 \mu \mathrm{m}$, and $250 \mathrm{~nm}$, respectively. $\theta$ is the angle from the surface normal in the plane perpendicular to the grating direction.

Figure 1 shows the calculated absorptance for $s$ and $p$ polarizations as a function of the inclination angle $\theta$ from the surface normal. We set the Cartesian coordinate system such that the $y$ axis is the direction in which the grating is ruled and the $z$ axis is normal to the surface. The inclination angle is in the $x z$ plane. The peak in the $p$ polarization is due to the surface phonon polaritons. The peak width is about $2.5^{\circ}$ and the approximate formula for the coherence length $l$

$$
l \sim \frac{\lambda}{\Delta \theta \cos \theta}
$$

yields $l \sim 32 \lambda$. However, the coherence calculated from Eq. (57) shown in Fig. 2 shows that the coherence length is much shorter. In Fig. 2, $\operatorname{Tr} \stackrel{\leftrightarrow}{W}^{(e)}$ is normalized to its value at $R_{x}=0$. The envelope of the normalized $\operatorname{Tr} \stackrel{\leftrightarrow}{W}^{(e)}$ decays to $1 / e$ at $\sim \lambda$. Thus the coherence length is $\sim \lambda$ even though the thermal emission is highly directional. This is partly due to the contributions of the modes that are not related to the surface phonon polaritons. These contributions are also manifested

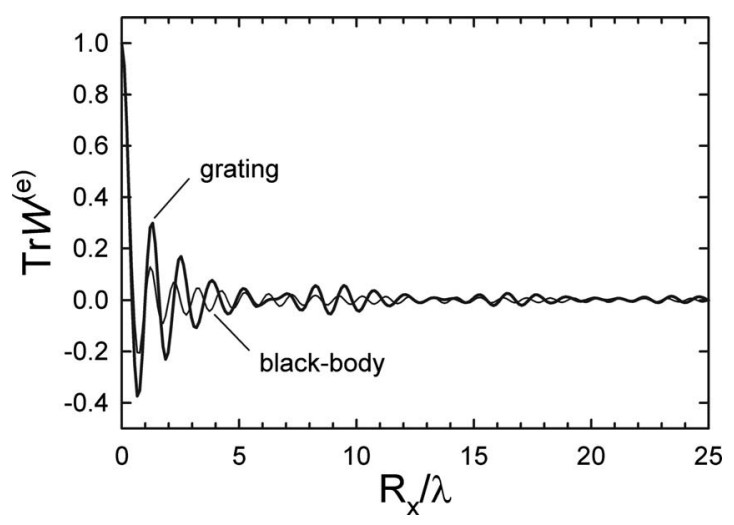

FIG. 2. Normalized electric coherence $\operatorname{Tr} \stackrel{\leftrightarrow}{W}^{(e)}$ as a function of the separation of the two points at $\lambda=11.36 \mu \mathrm{m}$ in the far field of the grating in Fig. 1. The distance between the two points $\left(R_{x}\right)$ is normalized to the wavelength $\lambda$. in the oscillating features with a periodicity of $\sim \lambda$ in Fig. 2 . These features are present in the $\operatorname{Tr} \stackrel{\leftrightarrow}{W}^{(e)}$ of a black body as shown as a dashed line in Fig. 2. However, this periodicity of $\sim \lambda$ is also contributed by the surface phonon polaritons. The peak in Fig. 1 at $\theta=46.7^{\circ}$ corresponds to $k_{x} a_{x} / 2 \pi=0.4$, where $a_{x}$ is the grating period. At this wave vector, the expected period in $\operatorname{Tr} \stackrel{\leftrightarrow}{W}^{(e)}$ is $R_{x} / \lambda=1.375$ from Eq. (57) if only the peak in Fig. 1 is considered. We also observe in Fig. 2 the beats with a period $R_{x} / \lambda \sim 9$. This cannot be explained by the surface phonon polariton modes in Fig. 1 or other modes different from surface phonon polaritons. The surface phonon polaritons couple to the light not only in the $x z$ plane but also in all the other planes perpendicular to the surface. These surface phonon polaritons have different wave vectors than $k_{\|} a_{x} / 2 \pi=0.4$ and contribute to the beats in Fig. 2. The exact locations of $\boldsymbol{k}_{\|}$can be found in Ref. 29. Because these $\boldsymbol{k}_{\|}$'s cover a broad range, this also contributes to the short coherence length in Fig. 2. Therefore, the large difference in the coherence length between Eq. (60) and Fig. 2 comes partly from the fact that Eq. (60) considers only the surface phonon polaritons in the $x z$ plane.

In Ref. 28, the coherence length of a grating shorter than that of a flat film was attributed to the radiative losses. Here, we find that the surface phonon polaritons with a range of wave vectors couple to the light for the grating and this is the main reason for the decrease in the coherence length. In this case, many different wave vectors are summed up in Eq. (57) resulting in dephasing which implies a decrease in the coherence length. The range of wave vectors is the consequence of the directional momentum transfer from the grating to the surface phonon polaritons. If we consider only the surface phonon polaritons of the wave vectors in the $x$ direction, Eq. (57) yields $l \sim 32 \lambda$ which agrees with the prediction of Eq. (60) and is not very different from $l \sim 36 \lambda$ predicted for a flat film. When we considered all the surface phonon polariton modes excluding the background emission, the calculated coherence was $l \sim 5 \lambda$ (not shown). Therefore, we conclude that the main reason for the decrease in the coherence length for the grating is not the radiation damping but the momentum transfer in the $x$ direction from the grating. If the grating has a cylindrical symmetry as in a bull's eye pattern, the momentum transfer occurs in the radial direction. Further, if the resulting momentum of the light is such that $\boldsymbol{k}_{\|} \simeq 0$, the coherence length due to the surface polaritons does not decrease by the momentum transfer because a very narrow range of wave vectors is involved. In this case, the light due to the surface polaritons will be beaming in the direction of surface normal. ${ }^{30}$

The calculation of coherence in the near field can be demanding even for simple structures. This is because large parallel wave vectors contribute to the coherence significantly in the near field. To see this more clearly, we note that Eq. (54) contains a factor $\exp \left[-k_{z I, v} z\right]$. From Eq. (17), $k_{z I, v}\left(\boldsymbol{k}_{\|}+\boldsymbol{g}_{\|}, \omega\right)$ increases as $\left|\boldsymbol{k}_{\|}+\boldsymbol{g}_{\|}\right|$increases. When $z$ is not small, the contributions of large $\left|\boldsymbol{k}_{\|}+\boldsymbol{g}_{\|}\right|$'s become negligible because $\exp \left[-k_{z I, v} z\right]$ becomes small. Thus, in this case, we do not need large $\left|\boldsymbol{g}_{\|}\right|$'s in numerical calculations. However, when $z$ is small, we need to include many terms in the summation over $\boldsymbol{g}_{\|}$'s in Eq. (54), which leads to a large compu- 


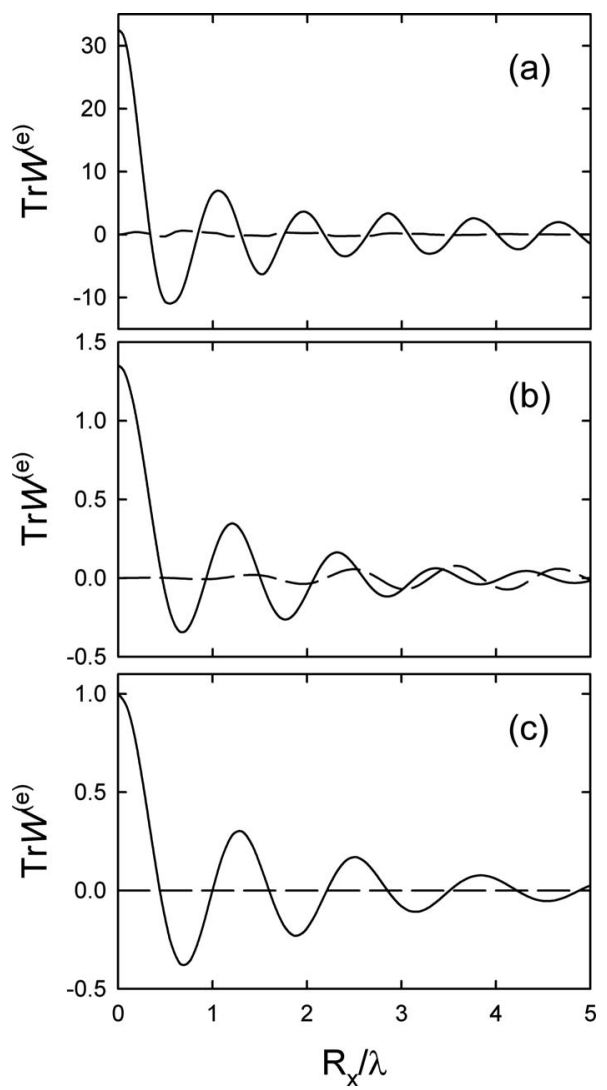

FIG. 3. Real (solid line) and imaginary (dashed line) part of the electric coherence $\operatorname{Tr} \stackrel{\leftrightarrow}{W}^{(e)}$ as a function of the separation of the two points at $\lambda=11.36 \mu \mathrm{m}$ in the near field at (a) $z=0.1 \lambda$, (b) $z=\lambda$, and (c) $z=10 \lambda$ of the grating in Fig. 1 . $\operatorname{Tr} \stackrel{\leftrightarrow}{W}^{(e)}$ 's are normalized to their value at $R_{x}=0$ and $z=10 \lambda$. The distance between the two points $\left(R_{x}\right)$ is normalized to the wavelength $\lambda$.

tation time. However, these calculations are suitable for a parallel computing approach because the calculations for each $\boldsymbol{g}_{\|}$can be done in parallel. Here we present calculations of optical coherence in the near field over a periodic structure. The grating used in the calculations is the same as the one in Fig. 1. In such structures with a mirror symmetry, the summation in $\boldsymbol{k}_{\|}$can be done only for $k_{x} \geq 0$. Because of a large computation time in the near-field calculations, we investigate the coherence on the planes at $z \geq 0.1 \lambda$.

Figure 3 shows $\operatorname{Tr} \overleftrightarrow{W}^{(e)}$ for the grating at $z=0.1 \lambda, \lambda$, and $10 \lambda$. All $\mathrm{Tr} \stackrel{\leftrightarrow}{W}^{(e)}$ 's are normalized to their values for $R_{x}=0$ at $z=10 \lambda$. At $z=10 \lambda$, the evanescent field is almost absent and the coherence is very similar to Fig. 2 . $\operatorname{Tr} \stackrel{\leftrightarrow}{W}^{(e)}$ is almost real at this far field. However, at $z=0.1 \lambda$ and $\lambda$, we observe that $\operatorname{Tr} \overleftrightarrow{W}^{(e)}$, which is real for a flat surface, is now complex. Moreover, the imaginary part does not necessarily decrease as $z$ increases even though it disappears for a sufficiently large $z$. The period of the oscillation in $\operatorname{Tr} \overleftrightarrow{W}^{(e)}$ decreases as $z$ decreases, which indicates that large wave vectors become important as the surface is approached. $W_{y y}^{(e)}$, which is associated with the fields polarized in the grating direction, behaves similar to the black body in the range of $z$ 's investigated (not shown). This indicates that the large coherence length in Fig. 3 is mainly due to the surface phonon polaritons which are $p$ polarized.

The electric energy density is proportional to $\operatorname{Tr} \stackrel{\leftrightarrow}{W}^{(e)}$ for $R_{x}=0$. In Fig. 3, we can see that the electric energy density increases as $z$ decreases. It is well known that, for a flat surface, the electric energy density decreases with increasing $z$ as $1 / z^{3}$ in the near field. ${ }^{17,18}$ However, this is not in general true for photonic crystals. To see this more clearly, we set $\boldsymbol{r}_{\| 1}=\boldsymbol{r}_{\| 2}$ and $z=z_{1}=z_{2}$ in Eq. (54) and define the electric energy density averaged over a unit cell $\bar{u}^{(e)}$ as

$$
\bar{u}^{(e)}(z, \omega)=\frac{1}{S_{u c}} \int_{u c} d \boldsymbol{r}_{\|} \sum_{l} W_{l l}^{(e)}\left(\boldsymbol{r}_{\|}, \boldsymbol{r}_{\|} ; z, z ; \omega\right) .
$$

Substitution of Eq. (54) for a uniform temperature into Eq. (61) yields

$$
\begin{aligned}
\bar{u}^{(e)}(z, \omega)= & \frac{\omega \mu_{0} \Theta}{4 \pi S} \sum_{\alpha, \beta} \sum_{\boldsymbol{k}_{\|}} \sum_{\boldsymbol{g}_{\|}} \frac{e^{-2 k_{z I, v}\left(\boldsymbol{k}_{\|}+\boldsymbol{g}_{\|}, \omega\right) z}}{\left|k_{z, v}\left(\boldsymbol{k}_{\|}+\boldsymbol{g}_{\|}, \omega\right)\right|} \\
& \times A^{(\alpha \beta--)}\left(-\boldsymbol{k}_{\|}-\boldsymbol{g}_{\|}, \omega\right),
\end{aligned}
$$

where the generalized absorptance $A^{(\alpha \beta--)}$ is defined similarly to Eq. (47) as

$$
\begin{aligned}
A^{(\alpha \beta--)}\left(-\boldsymbol{k}_{\|}-\boldsymbol{g}_{\|}, \omega\right)= & \frac{\omega}{c\left|k_{z, v}\left(\boldsymbol{k}_{\|}+\boldsymbol{g}_{\|}, \omega\right)\right| S_{u c}} \\
& \times \int d z^{\prime} \int_{u c} d \boldsymbol{r}_{\|}^{\prime} Q_{\left(-\boldsymbol{k}_{\|}-\boldsymbol{g}_{\|}, 0\right)}^{(\alpha \beta-)}\left(\boldsymbol{r}_{\|}^{\prime}, z^{\prime}, \omega\right)
\end{aligned}
$$

and the generalized absorption rate per unit volume $Q_{\left(-\boldsymbol{k}_{\|}-\boldsymbol{g}_{\|}, 0\right)}^{(\alpha \beta-)}$ is

$$
\begin{aligned}
Q_{\left(-\boldsymbol{k}_{\|}-\boldsymbol{g}_{\|}, 0\right)}^{(\alpha \beta-)}\left(\boldsymbol{r}_{\|}^{\prime}, z^{\prime}, \omega\right) & \\
= & \sum_{l, n} \frac{\omega}{c} \varepsilon_{I}\left(\boldsymbol{r}_{\|}^{\prime}, z^{\prime}, \omega\right) \\
& \times \frac{\widetilde{E}_{l}^{\alpha-*}\left(-\boldsymbol{k}_{\|}-\boldsymbol{g}_{\|}, 0, \omega\right) \widetilde{E}_{l}^{\beta-}\left(-\boldsymbol{k}_{\|}-\boldsymbol{g}_{\|}, 0, \omega\right)}{\widetilde{\boldsymbol{E}}^{\alpha-* 2}\left(-\boldsymbol{k}_{\|}-\boldsymbol{g}_{\|}, 0, \omega\right) \widetilde{\boldsymbol{E}}^{\beta-2}\left(-\boldsymbol{k}_{\|}-\boldsymbol{g}_{\|}, 0, \omega\right)} \\
& \times E_{-\boldsymbol{k}_{\|}\left(-\boldsymbol{k}_{\|}-\boldsymbol{g}_{\|,}, 0\right), n}^{(\alpha-n}\left(\boldsymbol{r}_{\|}^{\prime}, z^{\prime}, \omega\right) E_{-\boldsymbol{k}_{\|}\left(-\boldsymbol{k}_{\|}-\boldsymbol{g}_{\|}, 0\right), n}^{(\beta-)}\left(\boldsymbol{r}_{\|}^{\prime}, z^{\prime}, \omega\right) .
\end{aligned}
$$

$Q_{\left(-\boldsymbol{k}_{\|}-\boldsymbol{g}_{\|}, 0\right)}^{(\alpha \beta--)}$ is the local absorption rate per unit volume for the two sources that have polarizations $\alpha$ and $\beta$ and the same parallel wave vector $-\boldsymbol{k}_{\|}-\boldsymbol{g}_{\|}$at $z=0 . A^{(\alpha \beta--)}$ is the corresponding absorptance. When $1 / z \gg \omega / c$, it follows from Eq. (62) that

$$
\begin{aligned}
\bar{u}^{(e)}(z, \omega) \sim & \frac{\omega \mu_{0} \Theta}{16 \pi^{3}} \int_{0}^{\infty} d q_{\|} q_{\|} e^{-2 q_{\|} z} \\
& \times\left[\frac{\sum_{\alpha, \beta} \int_{0}^{2 \pi} d \phi A^{(\alpha \beta--)}\left(-\boldsymbol{q}_{\|}, \omega\right)}{\left|k_{z, v}\left(q_{\|}, \omega\right)\right|}\right],
\end{aligned}
$$

where $\boldsymbol{q}_{\|}=\boldsymbol{k}_{\|}+\boldsymbol{g}_{\|}$and $\phi$ is the azimuthal angle on the $x y$ plane. For a flat surface, the factor in the bracket in Eq. (65) 


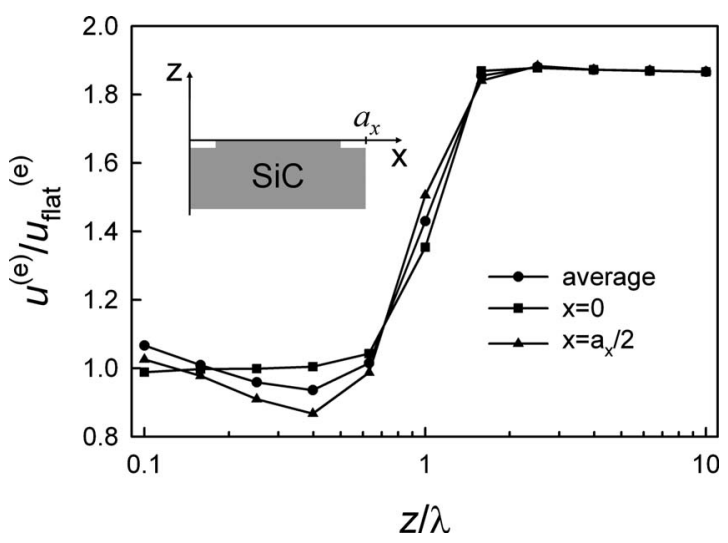

FIG. 4. The ratio of the electric energy density $u^{(e)}$ for the grating in Fig. 1 to $u_{\text {flat }}^{(e)}$ for the flat film as a function of the distance $z$ from the top of the grating at $\lambda=11.36 \mu \mathrm{m}$. The distance $z$ is normalized to the wavelength $\lambda . u^{(e)}$ was calculated at different positions in the $x$ direction. The coordinate system and the geometry of the unit cell are shown in the inset. $x$ is measured from the center of the groove. Square and triangular symbols are for $x=0$ and $x=a_{x} / 2=3.124 \mu \mathrm{m}$, respectively, and circular symbols show the average $\bar{u}^{(e)} / u_{\text {flat }}^{(e)}$ over the unit cell $0 \leq x \leq a_{x}$.

is proportional to $q_{\|}$and $\bar{u}^{(e)} \propto 1 / z^{3}$ in the near field. ${ }^{18}$ However, this does not happen in general for periodic structures. Therefore, the near-field heat transport can be modified when a uniform film is periodically structured.

The condition for $\bar{u}^{(e)} \propto 1 / z^{3}$ is typically satisfied for a flat surface when $z / \lambda<0.01 .{ }^{18}$ Due to the previously discussed long-time computation for the near field of photonic crystals in this range, we instead investigate the electric energy density behavior for $0.1 \leq z / \lambda \leq 10$. Figure 4 shows how the ratio of the electric energy density $u^{(e)}$ for the grating in Fig. 1 to $u_{\text {flat }}^{(e)}$ for the flat surface varies with the distance $z$ from the top of the grating. While the energy density of the flat surface is uniform on a plane parallel to the surface, at small distances from a corrugated surface it is inhomogeneous in the $x$ direction (see Fig. 4 inset for the coordinate system). As can be seen from Eq. (57), this inhomogeneity disappears in the far field. This is shown in Fig. 4. The distribution of the electric energy density in the $x$ direction depends on the distance $z$. For example, $u^{(e)}(x=0)$ is smaller than $u^{(e)}\left(x=a_{x} / 2\right)$ at $z / \lambda=1$ but becomes larger at $z / \lambda=0.4$. Overall, the energy density undergoes a significant change when $z \sim \lambda$ which marks the transition region between the near and the far fields. In the far field, the energy density of the grating is roughly twice that of the flat surface. However, the energy density averaged over the unit cell can be smaller or larger than the flat surface in the near field depending on the distance. These results imply that the distance dependence of heat transfer can be modified with periodic structures.

\section{CONCLUSION}

We have shown using fluctuational electrodynamics that thermal emission in the far field of photonic crystals obeys Kirchhoff's law. This does not assume that the photonic crystals are in thermal equilibrium with the radiation field but the material components should be in thermal equilibrium. Our proof is applicable to any photonic crystal structure. Thus, in the study of thermal emission, we can avoid the direct calculation of thermal emission from photonic crystals which is computationally intensive. ${ }^{13}$ Our formulation of fluctuational electrodynamics provides a comprehensive study of thermal emission from photonic crystals both in the far field and the near field. The coherence in the far field of a linear grating that supports surface polaritons is not much improved in comparison to the flat surface due to two reasons: (1) the directional momentum transfer from the grating to the surface polaritons and (2) the modes that are not related to the surface polaritons. These two lead to the far-field coherence length of $\sim \lambda$ which is not much different from the black body. The thermal near-field behavior of photonic crystals is shown to be different from a uniform film. This implies that near-field heat transport can be controlled by using photonic crystals.

\section{ACKNOWLEDGMENTS}

This work was supported by the U.S. Department of Energy (Grant No. DE-FG02-06ER46348) and used resources at the University of Minnesota Supercomputing Institute. The author thanks D. J. Norris and G. Chen for helpful comments, and the Samsung Foundation for financial support.

\footnotetext{
*Present address: Department of Mechanical Engineering, Massachusetts Institute of Technology, 77 Massachusetts Avenue, Cambridge, MA 02139, USA; sehan@mit.edu

${ }^{1}$ S. John and R. Wang, Phys. Rev. A 78, 043809 (2008).

${ }^{2}$ Y. De Wilde, F. Formanek, R. Carminati, B. Gralak, P. A. Lemoine, K. Joulain, J. P. Mulet, Y. Chen, and J. J. Greffet, Nature (London) 444, 740 (2006).

${ }^{3}$ S.-Y. Lin, J. Moreno, and J. G. Fleming, Appl. Phys. Lett. 83, 380 (2003).

${ }^{4}$ P. Nagpal, S. E. Han, A. Stein, and D. J. Norris, Nano Lett. 8, 3238 (2008).

${ }^{5}$ C. M. Cornelius and J. P. Dowling, Phys. Rev. A 59, 4736
}

(1999).

${ }^{6}$ J. G. Fleming, S.-Y. Lin, I. El-Kady, R. Biswas, and K. M. Ho, Nature (London) 417, 52 (2002).

${ }^{7}$ S. E. Han, A. Stein, and D. J. Norris, Phys. Rev. Lett. 99, 053906 (2007).

${ }^{8}$ J. D. Joannopoulos, P. R. Villeneuve, and S. Fan, Nature (London) 386, 143 (1997).

${ }^{9}$ T. Trupke, P. Wurfel, and M. A. Green, Appl. Phys. Lett. 84, 1997 (2004).

${ }^{10}$ S.-Y. Lin, J. Moreno, and J. G. Fleming, Appl. Phys. Lett. 84, 1999 (2004).

${ }^{11}$ I. El-Kady, W. W. Chow, and J. G. Fleming, Phys. Rev. B 72, 
195110 (2005).

${ }^{12}$ W. W. Chow, Phys. Rev. A 73, 013821 (2006).

${ }^{13}$ C. Luo, A. Narayanaswamy, G. Chen, and J. D. Joannopoulos, Phys. Rev. Lett. 93, 213905 (2004).

${ }^{14}$ J. G. Fleming, Appl. Phys. Lett. 86, 249902 (2005).

${ }^{15}$ C. H. Seager, M. B. Sinclair, and J. G. Fleming, Appl. Phys. Lett. 86, 244105 (2005).

${ }^{16}$ A. T. de Hoop, Appl. Sci. Res., Sect. B 8, 135 (1960).

${ }^{17}$ S. M. Rytov, Y. A. Kravtsov, and V. I. Tatarskii, Principles of Statistical Radiophysics, Vol. 3: Elements of Random Fields (Springer-Verlag, Berlin, 1989).

${ }^{18}$ C. Henkel, K. Joulain, R. Carminati, and J.-J. Greffet, Opt. Commun. 186, 57 (2000).

${ }^{19}$ C. T. Tai, Dyadic Green Functions in Electromagnetic Theory, 2nd ed. (IEEE, New York, 1994), Secs. 4 and 5.

${ }^{20}$ L. D. Landau and E. M. Lifshitz, Electrodynamics of Continuous Media, 2nd ed. (Pergamon, Oxford, 1984).
${ }^{21}$ J. E. Sipe, J. Opt. Soc. Am. B 4, 481 (1987).

${ }^{22}$ L. Mandel and E. Wolf, Optical Coherence and Quantum Optics (Cambridge University Press, Cambridge, 1995).

${ }^{23}$ L. D. Landau and E. M. Lifshitz, Statistical Physics, Part 1 (Pergamon, Oxford, 1984), Sec. 58.

${ }^{24}$ S. E. Han and D. J. Norris (unpublished).

${ }^{25}$ C. L. Mehta and E. Wolf, Phys. Rev. 161, 1328 (1967).

${ }^{26}$ J. B. Pendry and A. MacKinnon, Phys. Rev. Lett. 69, 2772 (1992).

${ }^{27}$ J.-J. Greffet, R. Carminati, K. Joulain, J. P. Mulet, S. Mainguy, and Y. Chen, Nature (London) 416, 61 (2002).

${ }^{28}$ F. Marquier, K. Joulain, J.-P. Mulet, R. Carminati, J.-J. Greffet, and Y. Chen, Phys. Rev. B 69, 155412 (2004).

${ }^{29}$ F. Marquier, C. Arnold, M. Laroche, J.-J. Greffet, and Y. Chen, Opt. Express 16, 5305 (2008).

${ }^{30}$ S. E. Han, P. Nagpal, and D. J. Norris (unpublished). 